\title{
Word associations and connected discourse: A reply to Lachman, Dumas and Guzy'
}

SHELDON ROSENBERG 2

GEORGE PEABODY COLLEGE FOR TEACHERS

In this article, the author replys to criticisms made of certain aspects of the interpretation and procedure of a study on associative factors in the recall of connected discourse. Methodological considerations and the results of new research lead the author to suggest again, that pre-experimental associative habits contributed to recall scores in the original study.

The high and low association passages employed by Rosenberg (1966a) in his study of associative factors in the recall of connected discourse contained stimulus and response words from word association norms and context. The context and the stimulus words were identical for both passages, while the response words differed in normative frequency. In this study, recall of stimulus and response words from the high association passage was found to be superior to recall of stimulus and response words from the low association passage.

Lachman, Dumas, \& Guzy (1966) have suggested that the context in question may have favored the high associative materials, and they present the results of a replication of Rosenberg's study with the addition of two control groups. In these groups, the context in the high and low association passages was randomized so as to "...destroy all syntactical structure, phrases and sentences."

In reply to Lachman, Dumas, and Guzy, the first point that should be made is that the task of their control groups may have been too difficult to expect to find a strong effect of associative strength after only a single, brief exposure. With instructions to try to remember "as much of the passage" as possible, the task of the control Ss was that of serial learning of a list of 149 words, presented in approximately $63 \mathrm{sec}$., in which strong syntactic and semantic constraints were violated. And as this consideration would have led one to anticipate, recall was generally lower in their control groups than it was in the groups that were exposed to the same words presented in a passage of connected discourse.

Clearly, if the control groups suggested by Lachman, Dumas, and Guzy are to be used, what appears to be needed is a study in which exposure interval, trials and instructions (recall for order vs. free recall) are varied. However, even with such a study, the control groups employed by Lachman, Dumas, and Guzy do not by any means remove the context factor; they merely replace one kind of context with another. A better control condition would be one in which all context was removed. In recent work (Rosenberg, 1966b) the stimulus and response words from high and low association passages were pulled out of context and presented as lists for learning in the order in which they appeared in their passages. In all instances in which this has been done, stimulus and response words from the high association passage were easier to learn than stimulus and response words from the low association passage.

Learning controls, of course, do not evaluate completely the possibility that context (in addition to associative habit) contributes something to recall. By deleting both the stimulus and the response words from a passage and asking Ss to guess at the missing items (cloze test), it is possible to estimate the degree of constraint exercised by the context. Recent data (Rosenberg, 1966b) from a study that employed a slightly modified version of the passages used in Rosenberg's (1966a) original study indicate that the context that Lachman, Dumas, and Guzy refer to predicts poorly the occurrence of stimulus and high and low strength response words. However, in the original study, Rosenberg obtained cloze data for the high and low association passages with either the stimulus or the response primaries (low strength counterparts in the case of the low association passage) deleted. Under these circumstances, "Guessing scores were clearly higher in the case of words deleted from the HA discourse." Thus, for example, if the stimulus words were deleted, the probability of their being guessed correctly was greater if the remaining passage contained high-strength responses than if it contained low-strength responses.

As a final point, it should be mentioned that Lachman, Dumas, and Guzy failed to find a significant main effect of associative strength for recall of the stimulus words. The reliability of this finding is questionable, since in all of the studies conducted to date (Rosenberg, 1966b) in our laboratory, recall of stimulus words from high association passages has been found to be significantly superior to recall of the same stimulus words embedded in low association passages. Such a finding cannot easily be attributed to possible facilitating effects of context, since the contexts in any given high and low association passages are identical.

With these considerations in mind, it seems reasonable to reiterate the suggestion made in the original study (Rosenberg, 1966a), namely, "... that associative habit plays an important role in the recall of connected discourse." 


\section{References}

Lachman, R., Dumas, J. S., \& Guzy, L. T. Joint effects of word association and approximation to English. Psychon. Sci., 1966, $5,145-146$.

Rosenberg, S. Associative factors in the recall of connected discourse. Psychon. Sci., 1966, 4, 53-54.
Rosenberg, S. Associative facilitation in the recall of connected discourse. Paper presented at the Conference on Verbal Learning ${ }^{*}$ Research and Written Instruction, New York, March, 1966.

\section{Notes}

1. The research referred to here was supported by Grant MH 08904 , from the National Institute of Mental Health, U. S. P. H. S.

2. Now at Center for Research in Language and Language Behavior, University of Michigan, Ann Arbor.

\section{Comment on Rosenberg's Reply to Lachman, Dumas, and Guzy}

\section{by Roy Lachman}

The complexity of natural language is such that when a variable is experimentally manipulated in the context of syntactic discourse, other variables are almost inevitably manipulated concomitantly. Thus, when Rosenberg inserted normatively high association words into an English paragraph, he modified the phrase content and very likely changed the transitional probabilities of the phrases in that paragraph.

An ideal design would involve orthogonal variation of word association level and natural language frequency of phrases in connected discourse. Since phrase frequencies are presently unavailable, Lachman, Dumas, and Guzy randomized the order of context words and thereby destroyed the phrase structure along with all syntax. The Lachman et al results demonstrate that word association and syntactical context interact to enhance recall, most likely because under these joint conditions the paragraph is loaded with high frequency phrases, cliches.

Rosenberg, in his reply, makes two main points. His first one is that the control groups were inappropriate as Ss had only 63 sec. to learn 149 unordered words. In point of fact, 63 sec. was sufficient for the high association control condition to show superior retention for all dependent variables employed except stimulus words (see Table 1 in Lachman et al). His final point was that Lachman et al failed to find a significant main effect for recall of stimulus words and "tine reliability of this finding is questionable." It is indeed questionable for, as Table 1 in Lachman et al shows, there is a reversal in the recall of stimulus words between the syntactical and nonsyntactical condition. The nonsignificant main effect, thus, is due to a very strong interaction. 